\title{
Avulsion and Replantation in Primary Dentition - A Review
}

\author{
Viddyasagar Prabhakar Mopagar¹, Meghana Vasant Phadnis², Sourabh Ramesh Joshi³ , \\ Vikranth Shetty ${ }^{4}$, Gowri Swaminatham Pendyala ${ }^{5}$ \\ 1, 2, 3 Department of Paediatric \& Preventive Dentistry, Rural Dental College, Pravara Institute of Medical \\ Sciences, Loni, Maharashtra, India. ${ }^{4}$ Department of Orthodontics, Tatyasaheb Kore Dental College and \\ Research Centre, New Pargoan, Kolhapur, Maharashtra, India. ${ }^{5}$ Department of Periodontology, Rural \\ Dental College, Pravara Institute of Medical Sciences, Loni, Maharashtra, India.
}

\section{ABSTRACT}

Avulsion of a primary tooth is a disturbing type of traumatic injury occurring in children. Paediatric dentists are likely to encounter a child with an avulsed primary tooth routinely in their clinical practice. Tooth avulsions are common in permanent teeth. However, avulsions can be seen even in primary teeth. The question of replantation of the avulsed tooth has been a focus of debate and controversy since years. The primary and most important factor in these injuries is to calculate the riskbenefit ratio keeping the underlying permanent tooth in mind. Utmost importance has to be given on the care of development and normal eruptive movements of the succedaneous tooth. This is mandatory if any treatment like replantation is to be done. The amount of primary root resorption, stage of development of the underlying permanent tooth, type of splinting to be done etc. needs a lot of attention. Many authors have given their opinions regarding the replantation of primary teeth based on the type of tooth avulsed. Moreover, the evidence might differ as to whether or not replantation might alter the difficulty levels for the eruption process of succedaneous tooth. There is not much evidence regarding the benefits and risks of replantation post avulsion of primary teeth. However, individual authors have reported a successful outcome in their case reports. This article reports a review on avulsed primary teeth replantation. This would enable the dentists to analyze the risks and benefits associated with replantation, thereby guiding them to arrive at the best clinical decision.

\section{KEY WORDS}

Primary Tooth, Avulsion, Replantation.
Corresponding Author: Dr. Meghana Phadnis,

Department of Paediatrics and Preventive Dentistry,

Rural Dental College,

Pravara Institute of Medical Sciences,

Loni, Bk, Maharashtra, India.

E-mail:meghs.p4@gmail.com

DOI: $10.14260 /$ jemds/2021/133

How to Cite This Article:

Mopagar VP, Phadnis MV, Joshi SR, et al. Avulsion and replantation in primary dentition - a review. J Evolution Med Dent Sci 2021;10(09):619-623, DOI: $10.14260 /$ jemds/2021/133

Submission 05-11-2020,

Peer Review 01-01-2021,

Acceptance 07-01-20201

Published 01-03-2021.

Copyright (C) 2021 Viddyasagar Prabhakar Mopagar et al. This is an open access article distributed under Creative Commons Attribution License [Attribution 4.0 International (CC BY 4.0)] 


\section{BACKGROUND}

In the growing age, children experience many dental traumatic episodes that can lead to injuries. Such episodes may have a wide range of problems, from minor to severe, and sometimes even life-threatening, depending on the force of impact during the trauma. Facial trauma in children primarily comprise of dentoalveolar injures caused by an external impact on the dentition and its surrounding area. Such trauma can give rise to dentoalveolar fractures, displacement or avulsion of teeth, thereby causing harm to a child's appearance, psychology and function. ${ }^{1}$ Studies have shown that avulsion of primary teeth comprises between $5.8 \%^{2}$ and $19.4 \%{ }^{3}$ of all types of traumatic injuries and $19.2 \%$ of luxation injuries, ${ }^{4}$ to the primary dentition. These are severe injuries as the force sustained upon the tooth is strong enough to displace the tooth from its respective socket completely. This disturbs the blood supply of the pulp, thereby exposing the cells of the periodontal ligament to the external environment.5,6,7 Primary teeth injuries are common in young children due to their lack of muscle coordination in the early years of life. The highest incidence of trauma in primary dentition occurs at the age of 1 to 3 years, when a child's motor coordination is developing, and the child learns to walk and run. ${ }^{8}$ Most injuries to primary teeth occur secondary to falls, followed by accidents, fights or violence, sports and child abuse, $, 9,10$ where the most commonly involved tooth is maxillary primary central incisor followed by maxillary lateral incisors and mandibular central incisors. ${ }^{3}$ The reason behind this might be the forward placement of maxillary anteriors due to increase over jet. Although, there are reports of avulsion of canines and molars, the incidences are very low. 11,12

Treatment of avulsion injuries in primary dentition has been a controversial topic since years. Available options for treatment are; a) no treatment, b) replacement of tooth and c) replantation of the avulsed tooth. ${ }^{11,13}$ Successful replantation of an avulsed tooth in permanent dentition debate the possibility of performing replantation in the primary dentition. Several factors, such as maintenance of balanced occlusion, development of speech, physiological aspects of mastication and effects on child psychology, ${ }^{9}$ favour replantation. However, the recent guidelines given for the treatment of traumatic dental injuries in the primary dentition do not recommend replantation of an avulsed primary tooth. Based on the risks factors like damaging the permanent tooth germ due to additional infections or inflammations, or ankylosis, ${ }^{14,15}$ many authors counter-indicate the replantation of primary tooth. Also, concerns regarding child's co-operation to a technique-sensitive procedure like replantation, are expressed. Hence, before recommendation for replantation of an avulsed primary tooth, the Federal University of Santa Catarina (UFSC) protocols ${ }^{16,17}$ demand some important considerations. These include the following: the strategic value of the primary tooth in the oral cavity, integrity of the alveolar bone, total extra-alveolar time, the contamination level of the location from where the tooth fell, storage media in which the tooth is kept while out of the alveolus, the presence of adjacent teeth to splint, and nutritious or nonnutritious habits in the child's routine that may affect the stability of the replanted tooth. Also, the avulsed primary tooth requires a subsequent endodontic treatment to avoid apical consequences of pulp necrosis. ${ }^{7,9}$
Till date, there is barely any light shed on replantation of an avulsed primary tooth, as most articles published are case reports offering variable outcomes. Also, it is unclear whether the harmful consequences are due to replantation, a combination of avulsion and replantation or impact during the injury. This article is aimed to perform a review of the literature and case series that are available on replantation of the avulsed primary teeth, and to offer an analysis of the risks and benefits, to guide dentists regarding the best clinical decisions that could be made in such cases.

\section{TREATMENT PLANS FOR AN AVULSED PRIMARY TOOTH}

The most commonly used treatment methods for an avulsed primary tooth are, $11,13,18-20$

- No treatment: The socket is cleaned with physiologic saline and left empty. ${ }^{18}$

- Replacement of avulsed teeth: The avulsed tooth is replaced with fixed anterior prosthetic appliance or with all-porcelain resin-bonded retainers ${ }^{20}$ or with a removable prosthetic appliance. (However, children at an early age are not co-operative to deal with a removable appliance).

- $\quad$ Replantation of the avulsed tooth. ${ }^{11,13}$

Treatment Protocols followed for replantation of an avulsed primary tooth.

While replanting the avulsed primary tooth, one must follow protocols similar to the one followed for permanent avulsed tooth. Following are some of the considerations to be kept in mind.

First and foremost, should be the stage of root development, to ensure the strategic value of the tooth. If the avulsed primary tooth shows any clear sign of physiological root resorption, the paediatric dentists should not go ahead with replantation of such a tooth. ${ }^{7}$ In addition to above mentioned point, the extra-oral time that has passed until the child reports to the dentist must be considered. According to several authors, maximum extra-oral time recommended for the replantation of a primary tooth should be 30 minutes. Minimising the extra-oral time would help achieve the survival of the periodontal ligament and removal of the clot from the tooth socket, which is still fluid, by irrigation with sterile saline solution. ${ }^{7,9,13,21,22}$ Authors have claimed that a major barrier to replantation could be the trapped clot in the socket which presents post trauma. The clot can also be pushed against the succedaneous permanent tooth bud; however, it may perhaps lead to morphological changes if any. ${ }^{22,23}$

Another important factor is the storage media for the tooth during its extra-oral time. The medium needs to maintain the viability of the root cells. HBSS (Hank's Balanced Salt Solution), physiologic saline solution or milk or child's own saliva or blood $^{7,9,21,24}$ are some of the suggested and most accepted storage media. Next important consideration is the degree of contamination. ${ }^{7}$ Both, the tooth and replantation area, should be kept as clean as possible to minimize the rate of infection and obtain a better prognosis. ${ }^{3,25}$ The Dental Trauma Guide has recommended the cleaning of the root surface and apical foramen with a stream of normal saline and soaking the tooth in saline, thereby removing contamination and dead cells from the root surface. Also, irrigation of the socket with saline is a 
must. Gingival lacerations should be sutured if necessary. Replantation should be performed with gradual, rhythmic movements, and the direction should always be apical to allow the dispersion of pressure through the Haversian system of the sockets' alveolar bone. ${ }^{7}$ Repositioning should be done by thumb and finger, as described by Andreasen. ${ }^{6}$

Avulsion injuries disrupt vascular nerve bundles, thereby restricting blood supply to the tooth. This can result in pulp necrosis, 5,6,7 extending to external inflammatory root resorption. Furthermore, maintenance of inflammation could give rise to an abscess with or without fistula, thus further contributing to the failure of replantation. Hence, a number of authors have recommended endodontic treatment of the avulsed tooth on which the prognosis of replantation is largely dependent. There has been a varied opinion as to time at which the endodontic treatment should be initiated. Studies have recommended this to be done in the very next appointment following replantation, with periodical exchange of intracanal medicament and subsequent root canal filling material and monitoring procedures. 7,9,16,17,26-28 This approach has given a remarkable outcome. Completion of root canal treatment, extra-orally in the avulsed tooth, before replantation has also reported success. ${ }^{26}$ Reports also proposed a successful outcome, with the resection of the root apex to about one fourth to one-fifth of the total root length followed by retrograde filling of the primary tooth root with calcium hydroxide. ${ }^{27}$ Case reports have shown replantation and immobilization of the avulsed primary tooth for $4-6$ weeks without carrying endodontic treatment at all.3,29 However, this could result in pulp necrosis and inflammatory root resorption, thereby affecting the apical bone tissue. ${ }^{7}$

Most case reports mentioned splinting of the replanted tooth.7,9,25,23,27-30 International Association of Dental Traumatology advice to place a flexible type of splint. This allows the physiological movement of the tooth, thus favouring reattachment of the periodontal ligament, thereby avoiding the risk of aspiration.9,31,32 Existence of neighbouring healthy teeth that would allow the placement of a splint is also an important factor to be taken into consideration. Ideally, splinting is recommended for 14 or 21 days. ${ }^{27,33}$

Antibiotics should be prescribed depending upon the degree of contamination. A soft diet should be advised for a minimum of one week. The follow-up period inclusive of clinical and radiographic evaluations, is essential. This ensures the success rate of replantation and allows the early detection of any signs of failure. ${ }^{7}$

\section{DISCUSSION}

There is an absence of scientific evidence supporting replantation of avulsed primary teeth in textbooks and literature. Even the recent guidelines of the International Association of Dental Traumatology and American Association of Paediatric Dentistry ${ }^{18}$ do not recommend replantation of primary teeth due to the potential damage to the developing permanent tooth germ. There are only case reports by various authors, purely based on reports of clinical cases, expert opinion and review articles, stating protocols for treatment of traumatized primary teeth. ${ }^{31}$ The above review aims to describe the probability of replantation of an avulsed primary tooth. According to the UFSC protocol and the studies conducted by authors such as Kinoshita et al.,9 Mueller \& Whitsett, ${ }^{29}$ Weiger \& Heuchert ${ }^{28}$, and Zamon ${ }^{34}$, replantation of the primary avulsed tooth was a recommended plan of treatment.

Many obvious advantages speak in favour of replantation of the avulsed primary teeth, as an acceptable treatment strategy. 1. Replantation helps in prevention of articulation problems: Early loss of maxillary primary anteriors before the age of four years could possibly distort the continuant consonants ( $\mathrm{v}, \mathrm{f}, \mathrm{th}, \mathrm{z}$, and $\mathrm{s})$. The phonetics for these continuant consonants requires forcing of the air stream through an opening, small enough in the oral cavity to produce friction noises. Hence, the presence or absence of primary incisor teeth plays a major role in the proper production of speech. ${ }^{35}$ 2. Space loss and effect on the eruption pathway: Due to a tendency of unintentional drifting of adjacent teeth into the edentulous space, intra-arch discrepancies can occur. This may result in the delay or ectopic eruption of succedaneous teeth with a resultant malocclusion. It could also affect the timing or path of eruption of the successive teeth ${ }^{36}$. 3 . Effect on oral habits: Premature loss of primary anterior teeth could act as an aetiologic factor for the development of tongue thrusting habit. ${ }^{36,37}$ 4. Mastication: Primary incisor teeth play an important role in the masticatory function of the child which can directly affect the child's nutritional status.

Despite the many justified benefits in favour of replantation of avulsed primary teeth, arguments have been raised, in textbooks, literature, reviews and guidelines for the management of trauma to primary teeth, against the process. Some of the most commonly proposed arguments are -

- Children have no aesthetic demand as they are not aware of the loss of primary incisor till the age of 5 or 6 , as stated by Moss: 38

- Damage to the permanent successors, $14,18,34,36,39$ Andreasen stated that damage to the permanent successor might be a result of the coagulum which is forced into the area of the follicle during insertion of the avulsed primary incisor back into its socket. ${ }^{40}$ This can be taken care off by ensuring complete removal of the blood clot by thoroughly rinsing it with physiologic saline. Many cases with a history of avulsion of primary teeth have reported damage to the permanent successor even without performing replantation procedure. ${ }^{3}, 23,41-44$

- Pulpal necrosis: Authors have stated the risk of pulpal necrosis followed by external root resorption, $6,45,46$ which could further give rise to abscess and fistulae. There is always a postulated risk when a probable source of infection is left in the tooth socket post avulsion. Hence, it is justified that endodontic treatment is a must in order to avoid future failures with replantation.

- Ankylosis: Several articles mention ankylosis as a complaint associated with replantation of primary teeth. 13,47,48 However, there are no confirmed statements reporting the same, thereby keeping the situation hopeful. According to Levine, ankylosis of replanted primary teeth occurs when the root has been stripped of its periodontal membrane, thereby, allowing joining of the cementum and the bone. ${ }^{47}$ Also, the extraoral time and the storage media in which the tooth was kept before replantation are important factors contributing to ankylosis. The viability of periodontal ligament is the key factor concerning results of replantation; as ankylosis of teeth post-traumatic injuries occur when osteoclasts and 
subsequently osteoblasts originating from the surrounding alveolar bone reach the root surface once they have crossed the damaged periodontal ligament (PDL) and precementum region. ${ }^{47,49}$ In short, ankylosis can occur following all types of injuries to the supporting structures. ${ }^{6}$ Hence, one might forecast ankylosis as a complication, however, there are no affirmations underlying the same.

- $\quad$ Risk of aspiration: Killian, Kawashima \& Pineda ${ }^{21}$ have expressed the risk of aspiration following replantation. Nevertheless, this risk was expressed because of not splinting the replanted tooth. This can be taken care of by providing a proper semi rigid type of splint with the help of adjacent unaffected teeth till the time the tooth appears stable.

- Child cooperation: Treatments like replantation of an avulsed tooth demand for high-level child co-operation. Concerns about lack of child cooperation also have been expressed as children until the age of around four years do not usually show a positive behaviour towards such technique sensitive procedures.

\section{CONCLUSIONS}

Preservation and replantation of avulsed primary teeth are a real challenge to paediatric dentists. There is a lack of substantial evidence, both, in favour of or against replantation and all arguments supporting or dismissing this procedure are based on sporadic case reports. No experimental studies demonstrating the damage to a permanent successor on account of replantation of an avulsed primary tooth have been published.

However, this does not mean that risks do not exist. It is presently not possible to consider replantation of an avulsed primary tooth as evidence-based care; thus, the procedure cannot be formally suggested. Consequently, it is advisable to apply a 'look for evidence' attitude, then vouching for refutation based on anecdotal evidence from statements that are published.

To conclude, it is crucial to consider all the advantages and the risk factors, follow appropriate protocols and abide by the requirements of favourable conditions. Keeping this in mind, dentists can decide whether or not to go ahead with replantation of an avulsed primary tooth.

Financial or other competing interests: None.

Disclosure forms provided by the authors are available with the full text of this article at jemds.com.

\section{REFERENCES}

[1] Borum MK, Andreasen JO. Sequelae of trauma to primary maxillary incisors. I. Complications in the primary dentition. Endod Dent Traumatol 1998;14(1):31-44.

[2] Kenwood M, Seow WK. Sequelae of trauma to the primary dentition. J Pedod 1989;13(3):230-8.

[3] Ravn JJ. Sequelae of acute mechanical traumata in the primary dentition. A clinical study. ASDC J Dent Child 1968;35(4):281-9.
[4] Soporowski NJ, Allred EN, Needleman HL. Luxation injuries of primary anterior teeth--prognosis and related correlates. Pediatr Dent 1994;16(2):96-101.

[5] American Academy on Pediatric Dentistry Council on Clinical Affairs. Guideline on management of acute dental trauma. Pediatr Dent 2008;30(7 Suppl):175-83.

[6] Brandt RS. [Andreasen JO, Andreasen FM. Textbook and colour atlas of traumatic injuries to the teeth. $3^{\text {rd }}$ edn. Copenhagen: Munksgaard 1993: p. 762. Hardback, DKK 1400.00. ISBN 871610637 7]. Journal of Dentistry June 1995;23(3):191.

[7] de Carvalho Rocha MJ, Cardoso M. Reimplantation of primary tooth--case report. Dent Traumatol 2008;24(4):e4-10.

[8] Onetto JE, Flores MT, Garbarino ML. Dental trauma in children and adolescents in Valparaiso, Chile. Endod Dent Traumatol 1994;10(5):223-7.

[9] Kinoshita S, Mitomi T, Taguchi Y, et al. Prognosis of replanted primary incisors after injuries. Endod Dent Traumatol 2000;16(4):175-83.

[10] Andreasen J0. Etiology and pathogenesis of traumatic dental injuries. A clinical study of 1,298 cases. Scand J Dent Res 1970;78(4):329-42.

[11] Steelman R, Holmes D, Byron M, et al. Traumatic avulsion of the mandibular right primary lateral incisor and cuspid. J Clin Pediatr Dent 1991;15(4):249-50.

[12] Kupietzky A. The treatment and long-term management of severe multiple avulsion of primary teeth in a 19months-old child. Pediatr Dent 2001;23(6):517-21.

[13] Crabb JJ, Crabb VP. Reimplantation of a primary central incisor: a case report. Dent Pract Dent Rec 1971;21(10):353-4.

[14] Fried I, Erickson P. Anterior tooth trauma in the primary dentition: incidence, classification, treatment methods, and sequelae: a review of the literature. ASDC J Dent Child 1995;62(4):256-61.

[15] Harding AM, Camp JH. Traumatic injuries in the preschool child. Dent Clin North Am 1995;39(4):817-35.

[16] Cardoso M, de Carvalho Rocha MJ. Federal University of Santa Catarina follow-up management routine for traumatized primary teeth - part 1 . Dental Traumatology 2004;20(6):307-13.

[17] de Carvalho Rocha MJ, Cardoso M. Federal University of Santa Catarina endodontic treatment of traumatized primary teeth - part 2. Dental Traumatology 2004;20(6):314-26.

[18] Malmgren B, Andreasen JO, Flores MT, et al. International Association of Dental Traumatology guidelines for the management of traumatic dental injuries: 3 . Injuries in the primary dentition. Dental Traumatology 2012;28(3):174-82.

[19] Aejaz A. Modified gropers appliance for enhanced esthetics: a case theme. International Journal of Advanced Research 2019;7(9):254-8.

[20] Huber CT. Resin-bonded retainer for replacement of an avulsed primary incisor: a case report. Quintessence Int 1997;28(5):337-9.

[21] Kawashima Z, Pineda FR. Replanting avulsed primary teeth. J Am Dent Assoc 1992;123(10):90-1, 94.

[22] Bassiouny MA, Giannini P, Deem L. Permanent incisors traumatized through predecessors: sequelae and possible management. J Clin Pediatr Dent 2003;27(3):223-8. 
[23] Christophersen P, Freund M, Harild L. Avulsion of primary teeth and sequelae on the permanent successors. Dent Traumatol 2005;21(6):320-3.

[24] Pefaur A. Reimplantation of a deciduous tooth after traumatic avulsion. Rev Fac Odontol Univ Chile 1988;6(1):20-4.

[25] Gatewood JC, Thornton JB. Successful replantation and splinting of a maxillary segment fracture in the primary dentition. Pediatr Dent 1995;17(2):124-6.

[26] Eisenberg MD. Reimplantation of a deciduous tooth. Oral Surgery Oral Medicine Oral Pathology Oral Radiology 1965;19(5):588-90.

[27] Filippi A, Pohl Y, Kirschner H. Replantation of avulsed primary anterior teeth: treatment and limitations. ASDC J Dent Child 1997;64(4):272-5.

[28] Weiger R, Heuchert T. Management of an avulsed primary incisor. Dental Traumatology 1999;15(3):138-43.

[29] Mueller BH, Whitsett BD. Management of an avulsed deciduous incisor. Oral Surgery, Oral Medicine, Oral Pathology Oral Radiol 1978;46(3):442-6.

[30] Flores MT, Malmgren B, Andersson L, et al. Guidelines for the management of traumatic dental injuries. III. Primary teeth. Dent Traumatol 2007;23(4):196-202.

[31] Friedlander LT, Chandler NP, Drummond BK. Avulsion and replantation of a primary incisor tooth. Dent Traumatol 2013;29(6):494-7.

[32] Holan G. Replantation of avulsed primary incisors: a critical review of a controversial treatment. Dent Traumatol 2013;29(3):178-84.

[33] Acharya S, Mohanty S, Panigrahi A, et al. Avulsion and replantation of primary teeth - a feasible option. Dentist Case Rep 2017;1(1):1-3.

[34] Zamon EL, Kenny DJ. Replantation of avulsed primary incisors: a risk benefit assessment. J Can Dent Assoc 2001;67(7):386.

[35] Kalia G, Tandon S, Bhupali NR, et al. Speech evaluation in children with missing anterior teeth and after prosthetic rehabilitation with fixed functional space maintainer. Journal of Indian Society of Pedodontics and Preventive Dentistry 2018;36(4):391-5.

[36] Holan G, Needleman HL. Premature loss of primary anterior teeth due to trauma - potential short- and longterm sequelae. Dental Traumatology 2014;30(2):100-6.
[37] Wright GZ, Friedman CM. Management of dental trauma In: Braham RL, Morris M, eds. Textbook of Pediatric Dentistry. $2^{\text {nd }}$ edn. Baltimore: Williams \& Wilkins 1985.

[38] Moss SJ, Maccaro H. Examination, evaluation and behavior management following injury to primary incisors. NY State Dent J 1985;51(2):87-92.

[39] Nelson LP, Shusterman S. Emergency management of oral trauma in children. Curr Opin Pediatr 1997;9(3):242-5.

[40] Andreasen JO, Andreasen FM. Injuries to the primary dentition. Chap - 10. In: Essentials of traumatic injuries to the teeth. $1^{\text {st }}$ edn. Copenhagen: Munksgaard 1990: p. 14154.

[41] Andreasen J0, Ravn JJ. The effect of traumatic injuries to primary teeth on their permanent successors. II. A clinical and radiographic follow-up study of 213 teeth. European J Oral Sciences 1971;79(3):284-94.

[42] Brin I, Fuks A, Ben-Bassat Y, et al. Trauma to the primary incisors and its effect on the permanent successors. Pediatr Dent 1984;6(2):78-82.

[43] Tewari N, Pandey RK. Root hypoplasia: an unusual sequela to primary tooth trauma. Dent Traumatol 2010;26(1):115-7.

[44] Ravn JJ. Developmental disturbances in permanent teeth after exarticulation of their primary predecessors. Scand J Dent Res 1975;83(3):131-4.

[45] Andreasen J0. Relationship between surface and inflammatory resorption and changes in the pulp after replantation of permanent incisors in monkeys. J Endod 1981;7(7):294-301.

[46] Cully JL, Zeeb K, Sahay RD, et al. Prevalence of primary teeth injuries presenting to a pediatric emergency department. Pediatric Dentistry 2019;41(2):136-9.

[47] Levine N. Injury to the primary dentition. Dent Clin North Am 1982;26(3):461-80.

[48] McDonald RE, Avery DR, Dean JA, et al. Management of trauma to the teeth and supporting tissues. McDonald and Avery Dentistry for the Child and Adolescent 2011: p. 403-42.

[49] Lindskog S, Pierce AM, Blomlof L, et al. The role of the necrotic periodontal membrane in cementum resorption and ankylosis. Endod Dent Traumatol 1985;1(3):96-101. 